\title{
Evaluation of volumetric modulated arc therapy (VMAT) - based total body irradiation (TBI) in pediatric patients
}

\author{
Kadir Yaray, Edrine Damulira \\ Department of Radiation Oncology, M.K. Dedeman Oncology Hospital, School of Medicine, Erciyes University, Kayseri, Turkey
}

\begin{abstract}
Background: The dosimetric characterization of volumetric modulated arc therapy (VMAT)-based total-body irradiation (TBI) in pediatric patients is evaluated.

Materials and methods: Twenty-two patients between the ages of 2 and 12 years were enrolled for VMAT-based TBI from 2018 to 2020 . Three isocenters were irradiated over three overlapping arcs. While prescribing $90 \%$ of the TBI dose to the planning treatment volume (PTV), two fractions (2 Gy each) were delivered each day; hence 12 Gy was delivered in six fractions. During treatment optimization, the mean lung and kidney doses were set not to exceed $7 \mathrm{~Gy}$ and $7.5 \mathrm{~Gy}$, respectively. The maximum lens dose was also set to less than $4 \mathrm{~Gy}$. Patient quality assurance was carried out by comparing treatment planning system doses to the 3-dimensional measured doses by the ArCCHECK ${ }^{\circledast}$ detector. The electronic portal imaging device (EPID) gamma indices were also obtained.

Results: The average mean lung dose was $7.75 \pm 0.18 \mathrm{~Gy}$, mean kidney dose $7.63 \pm 0.26 \mathrm{~Gy}$, maximum lens dose $4.41 \pm 0.39 \mathrm{~Gy}$, and the mean PTV dose $12.69 \pm 0.16 \mathrm{~Gy}$. The average PTV heterogeneity index was $1.15 \pm 0.03$. Average differences in mean kidney dose, mean lung dose, and mean target dose were $2.79 \% \pm 0.88,0.84 \% \pm 0.45$ and $0.93 \% \pm 0.47$, respectively; when comparing planned and $\mathrm{ArCCHECK}^{\circledR}$ measured doses. Only grade 1-2 radiation toxicities were recorded, based on CTCAE v5.0 scoring criteria.
\end{abstract}

Conclusions: VMAT-TBI was characterized with good PTV coverage, homogeneous dose distribution, planned and measured dose agreement, and low toxicity.

Key words: toxicity; homogeneity; medical dosimetry; organs at risk (OARs); planned target volume (PTV)

Rep Pract Oncol Radiother 2021;26(4):518-527

\section{Introduction}

Cancers such as acute lymphoblastic leukemia (ALL) and acute myeloid leukemia (AML) attack and incapacitate blood generating cells in the bone marrow. Therefore, bone marrow transplantation becomes indispensable for patients with ALL and AML. However, after the bone marrow transplantation procedure, the immune system of the recipient could reject the donor's bone marrow. Thus, before the transplantation operation is executed, a conditioning regimen is prescribed to bone marrow recipients. The regimen is normally aimed at creating space for new bone marrow and also eliminates malignant cells, since they are radiosensitive [1]. In other terms, the conditioning regimen is an immunosuppression procedure [1]. The regimen could be implemented solely with chemotherapy (drugs), or in conjunction with radiation therapy, in the form of total body irradiation (TBI).

Address for correspondence: Kadir Yaray, Department of Radiation Oncology, M.K. Dedeman Oncology Hospital, School of Medicine, Erciyes University, 38039 Kayseri, Turkey; e-mail: yaray_2000@yahoo.com

This article is available in open access under Creative Common Attribution-Non-Commercial-No Derivatives 4.0 International (CC BY-NC-ND 4.0) license, allowing to download articles and share them with others as long as they credit the authors and the publisher, but without permission to change them in any way or use them commercially 
During TBI, the planned target volume (PTV) is the whole body. In this case, the dose delivered to the whole body (PTV) should be sufficient to achieve the goals of the plan, but also spare the organs at risk (OARs). Particularly, during TBI, the OARs mostly include the lungs, kidneys, the liver and pre-irradiated regions [2, 3]. Even with execution of optimum PTVs, TBI is associated with side effects including tiredness, nausea and vomiting, cataracts, skin redness, infertility in both men and women, hair loss, diarrhea, and even induction of secondary cancers. Owing to the those side effects, dose heterogeneities, toxicities, and secondary malignancies [4], there has been a significant decline in the TBI-based conditioning regimens [5].

Classical TBI is associated with shortcomings, such as longer treatment delivery times and higher toxicity levels, in the form of delivering non-sparing doses to OARs (in both pediatric and adult patients) $[6,7]$. Diverging from the 30-year-old conventional TBI execution techniques that employ non-conformal large opposed whole-body fields [2], current methods have resorted to implementing targeted TBI in the form of total marrow irradiation (TMI) [1]. TMI has been achieved using image-guided intensity modulated radiation therapy (IG-IMRT) [1]. Other studies have exploited volumetric modulated arc therapy (VMAT) for delivery of TMI $[1,3,8,9]$. This VMAT technique has been implemented in order to reduce organ doses and toxicities, and also improve dose uniformity [1]. During TMI treatment planning, 3-4 isocenters cover the target organs and the treatment is delivered in multiple dynamic IMRT arcs [1].

However, limited studies have reported the evaluation of the radiation doses and toxicities/morbidities associated with VMAT-based TMI/TBI in pediatric patients. Moreover, most clinical TMI trials recently done are pilot or phase one trials [1]. Furthermore, as observed in various studies $[8,10$, 11], pediatric and adult patients are prescribed the same dose, i.e., a total of $12 \mathrm{~Gy}$ (2 Gy per fraction), during treatment delivery. However, radiation exposure to pediatric patients should be stringent because they still have a longer lifespan to live. For instance, growth disturbances were reported in prepubertal pediatric patients, where two treated patients, identical twins, lost $8 \%$ of their height [6].
Owing to TBI related radiation exposures, children are at a high risk of developing multiple endocrine disorders [12]. For those reasons, in some clinics, cutting-edge and precise techniques employing proton therapy are reserved for/priority is given to pediatric patients.

Whereas studies have demonstrated VMAT could be a feasible technique for delivering TBI $[13,14]$, limited focus has been directed towards the implications and dosimetric characterization of VMAT-based TBI in pediatric patients.

In this investigation, VMAT plans are formulated in the treatment planning system (TPS) and executed using a linear accelerator, following the normal clinical workflow. While patient-specific quality assurance (QA) is carried out using the Arc$\mathrm{CHECK}^{\oplus}$ detector array and the electronic portal imaging device (EPID), the toxicity score is evaluated according to the Common Terminology Criteria for Adverse Events (CTCAE v5.0).

This study, therefore, aims at evaluating the appropriateness of VMAT-based TBI for pediatric patients, by elaborating the dosimetric characterization associated with this technique in pediatric patients.

\section{Materials and methods}

\section{Patient selection}

Pediatric patients in this study were stem-cell transplant candidates who received TBI treatment from our oncology hospital. Twenty-two patients at the ages of 2 to 12 years were enrolled into the study from 2018 to 2020 . Four were female, whereas 18 were male.

\section{Patient positioning during computed tomography (CT)}

The patients were laid on the patient couch of a Siemens (SOMATOM Emotion ${ }^{\mathrm{TM}}$, Erlangen, Germany) CT scanner in a supine position. For immobilization, a vacuum positioning cushion $\left(\mathrm{CIVCO}^{\circ}\right.$ Radiotherapy Vac-Lok ${ }^{\mathrm{TM}}$ \& Positioning Cushions, Auckland, New Zealand) was additionally placed on the patient couch.

\section{Contouring}

The Varian Eclipse SomaVision (Varian Medical Systems, Palo Alto, CA) software interface was employed for contouring. Each CT slice was $3 \mathrm{~mm}$. 
The contoured organs included lungs, kidneys, and lens. The PTV comprised the whole body, and a 2 $\mathrm{mm}$ margin was allowed between the PTV and the skin.

\section{Treatment planning and dose prescription}

The VMAT plans implemented for TBI in this study were created using the Varian TrueBeam v2.5 linear accelerator (Varian Medical Systems, Palo Alto, CA). This linac is equipped with a Varian Millenium 120-leaf multi-leaf collimator system (MLC) (Varian Medical Systems, Palo Alto, CA). At a nominal skin-axis distance $(\mathrm{SAD})$ of $100 \mathrm{~cm}$, the maximum field size was $40 \times 40 \mathrm{~cm}^{2}$, and MLC minimum leaf-width is $5 \mathrm{~mm}$.

The Eclipse ${ }^{\mathrm{TM}}$ TPS version 13.6 (Varian Medical Systems, Palo Alto, CA) was used to formulate dual-arc VMAT plans implemented here for TBI while executing the anisotropic analytical algorithm (AAA) 13.6.23 calculation configuration. The nominal variable dose rate was set to acquire a dose rate of $600 \mathrm{MU} / \mathrm{min}$ in the $50-600 \mathrm{MU} / \mathrm{min}$ range.

During treatment planning optimization, $90 \%$ of the PTV was prescribed a total dose of $12 \mathrm{~Gy}$ in six fractions. Two fractions (2 Gy each) were delivered to patients on three consecutive days. A time delay of at least six hours was observed between the fractions. The mean doses to the lungs and kidneys were set to be less than 7 Gy and $7.5 \mathrm{~Gy}$, respectively. The maximum lens dose was also set to be less than $4 \mathrm{~Gy}$. In the present study, we defined the heterogeneity index (HI) as the ratio of D5 to D95, where D5 and D95 are the minimum doses encompassing $5 \%$ and $95 \%$ of the PTV, respectively.

The linac executed these plans using the $6 \mathrm{MV}$ photon energy setting. This linac's Varian On-Board Imager $^{\mathrm{TM}}(\mathrm{OBI})$ kilovoltage imaging system ascertained the patient positioning preceding irradiations.

\section{Patient set-up verification}

Patient set-up was verified by $\mathrm{kV}$ cone-beam CT (CBCT) imaging (TrueBeam v2.5) incorporated in the linac's system, in the form of image-guided RT (IGRT) for the VMAT-TBI.

The average beamon time for each patient was $30 \pm 5$ minutes, whereas the average patient set-up and positioning time was 20 minutes.

\section{Quality assurance}

The pre-treatment VMAT-based plans for all the patients enrolled in this study were verified using the ArcCHECK $^{\oplus}$ (Sun Nuclear: Melbourne, FL, USA) four-dimensional (4-D) detector array and the EPID. The ArcCHECK ${ }^{\oplus}$ array system is in the form of a cylinder with a diameter of $21 \mathrm{~cm}$ which constitutes 1,386 SunPoint Diode detectors in a helical grid geometry ( $1 \mathrm{~cm}$ offset). This ArcCHECK ${ }^{\oplus}$ device's array detector spacing is $1 \mathrm{~cm}$ and it also consists of acrylic phantom material. The device was placed on the treatment couch as per the positioning guidelines in the product manual (one side facing the gantry). A field size of $10 \mathrm{~cm} \times 10 \mathrm{~cm}$ was set up on the detector array matrix. We calculated the percentage dose differences between the planned and measured mean kidney dose, mean lung dose, and mean PTV dose using the ArcCHECK ${ }^{\oplus}$ system.

Furthermore, before treatment delivery, additional treatment plan verification in the form of EPID-based gamma indices was executed. For each patient, the average of the gamma indices from each PTV was computed and tabulated.

\section{Treatment plan settings and optimization}

As in similar studies $[1,8]$, the dose delivery was implemented using three isocenters with dual arcs. The arc angles were clockwise from $181^{\circ}$ to $179^{\circ}$, and counterclockwise from $179^{\circ}$ to $181^{\circ}$. The overlapping regions of the arcs were up to $2 \mathrm{~cm}$ and were taken into account by the treatment planning system, in order to avoid double dose in the junction regions. Isocenter positions were automatically set along the patient's longitudinal axis by the Eclipse TPS, observed as red dots in Figure 1A. Each arc was irradiated using one isocenter, as shown in Figure 1B. The three arcs were consecutively irradiated while the treatment couch was longitudinally translated. During the execution of each arc, the collimator was also rotated to $10^{\circ}$.

Anatomic structures were automatically contoured using Varian's comprehensive automatic and semi-automatic contouring tools software - Varian Eclipse SomaVision. The automatically contoured structures were checked and manually corrected/adjusted if necessary. On average, contouring, planning and optimization required approximately 6 hours. This time includes discussions and evaluations by the oncologists and physicists. 
A

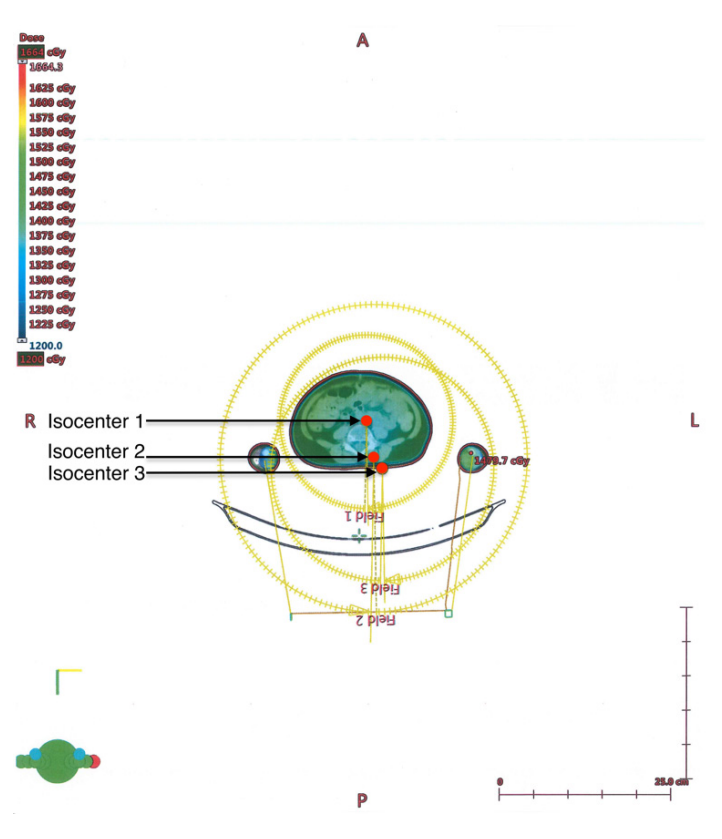

B

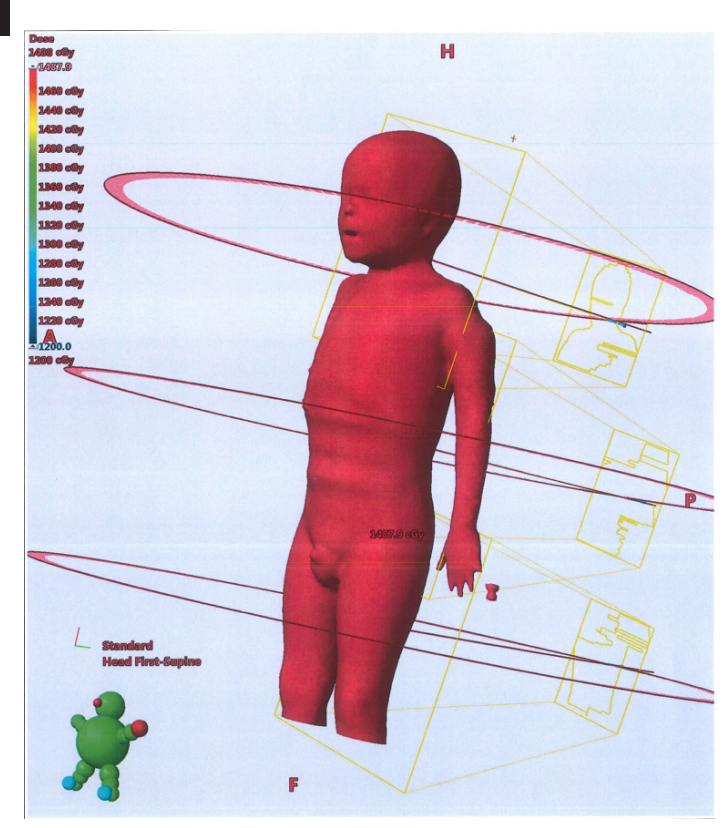

Figure 1. Isocenter positions (A) and arc ranges (B)

\section{Toxicity evaluation}

Investigation into the level of toxicity associated with the treatments was evaluated while referencing the CTCAE v5.0.

\section{Results}

\section{Dose distribution and patient QA}

A summary of the three-dimensional (3D) dose distributions in the lungs, clinical target volume (CTV), and PTV are graphically represented in the form of a dose volume histogram (DVH) in Figure 2.
Upon planned and ArcCHECK ${ }^{\oplus}$ system measured dose calculations, we obtained average differences of $0.84 \% \pm 0.45,2.79 \% \pm 0.88$, and $0.93 \% \pm 0.47$ in the mean lung, mean kidney, and mean PTV doses, respectively, as elaborated in Table 1.

Also, the mean EPID average PTV gamma index across all the 22 patients was 0.37 with a standard deviation of 0.028 , as observed in Table 1.

\section{Dosimetric characterization}

Among the 22 pediatric patients enrolled in this study, the average mean lung and kidney

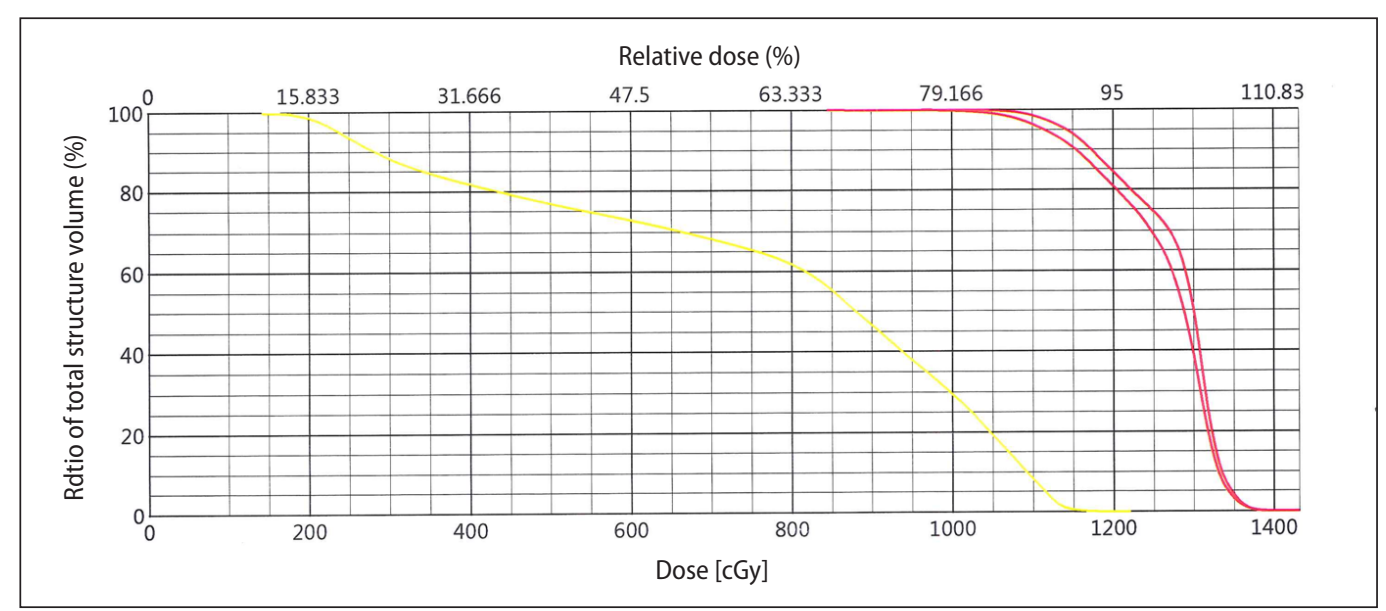

Figure 2. Dose volume histogram: yellow — lungs, inner red — planned target volume (PTV) — PTV, and outer red — clinical target volume (CTV) 
Table 1. ArcCHECK ${ }^{-}$-based dose differences and average planned target volume (PTV) $\gamma$-index per patient

\begin{tabular}{|c|c|c|c|c|}
\hline $\begin{array}{l}\text { Patient Number } \\
\text { (\#) }\end{array}$ & $\begin{array}{l}\text { Mean lung dose } \\
\text { difference (\%) }\end{array}$ & $\begin{array}{l}\text { Mean kidney dose } \\
\text { difference (\%) }\end{array}$ & $\begin{array}{l}\text { Mean PTV dose } \\
\text { difference (\%) }\end{array}$ & $\begin{array}{l}\text { EPID Average } \\
\text { PTV } \gamma \text {-index }\end{array}$ \\
\hline 1 & 0.7 & 2.4 & 0.6 & 0.39 \\
\hline 2 & 0.4 & 3.3 & 0.5 & 0.34 \\
\hline 3 & 0.3 & 1.7 & 0.6 & 0.38 \\
\hline 4 & 1.3 & 3.6 & 0.3 & 0.37 \\
\hline 5 & 0.2 & 1.9 & 1.1 & 0.41 \\
\hline 6 & 0.7 & 2.3 & 1.9 & 0.36 \\
\hline 7 & 1.1 & 2.6 & 0.6 & 0.33 \\
\hline 8 & 0.8 & 3.9 & 1.4 & 0.41 \\
\hline 9 & 0.6 & 2.2 & 0.8 & 0.35 \\
\hline 10 & 0.4 & 2.8 & 0.8 & 0.36 \\
\hline 11 & 1.1 & 2.0 & 1.2 & 0.37 \\
\hline 12 & 1.5 & 1.7 & 1.6 & 0.36 \\
\hline 13 & 0.5 & 3.1 & 0.9 & 0.33 \\
\hline 14 & 0.6 & 4.2 & 0.4 & 0.42 \\
\hline 15 & 1.7 & 2.8 & 1.0 & 0.39 \\
\hline 16 & 1.3 & 1.3 & 1.3 & 0.34 \\
\hline 17 & 0.2 & 2.2 & 0.6 & 0.32 \\
\hline 18 & 0.5 & 2.9 & 1.7 & 0.38 \\
\hline 19 & 0.8 & 2.4 & 0.5 & 0.36 \\
\hline 20 & 1.4 & 4.5 & 0.3 & 0.34 \\
\hline 21 & 1.3 & 2.7 & 1.4 & 0.40 \\
\hline 22 & 1.1 & 4.1 & 0.9 & 0.37 \\
\hline Mean \pm SD & $0.84 \pm 0.45$ & $2.79 \pm 0.88$ & $0.93 \pm 0.47$ & $0.37 \pm 0.028$ \\
\hline
\end{tabular}

SD — standard deviation

doses were 7.75 Gy and $7.63 \mathrm{~Gy}$, respectively. On the other hand, the average for the maximum lens dose and mean PTV dose were also 4.41 and $12.66 \mathrm{~Gy}$, respectively. The maximum lens dose was characterized with the highest percentage error in dose $(8.80 \%)$ whereas the PTV mean dose had the lowest percentage error $(1.27 \%)$, as can be observed in Table 2. Furthermore in Table 2, the average heterogeneity index (HI) of the PTV for all the 22 cases was $1.15 \pm 0.03$ and the average dose covering $95 \%$ of the target (D95) was also 11.4 Gy.

During treatment planning, the dose distribution attained by the TPS in the transverse, coronal and sagittal planes is illustrated in Figure 3. Upon execution of the treatment plans on the patients, the mean follow-up time of all the patients was 9 months.

When our VMAT-TBI plans were recalculated with a $5-\mathrm{mm}$ misalignment, on average; $4.0 \% \pm 1.6$ higher lung mean dose, $9.9 \% \pm 3.1$ higher lung max. dose, and $12.3 \% \pm 1.9$ higher PTV max. dose was attained. On the other hand, recalculation with a $10-\mathrm{mm}$ misalignment resulted in higher lung mean, lung max., and PTV max. doses which were on average; $6.2 \% \pm 1.7,15.0 \% \pm 3.8$, and $18.8 \% \pm 2.7$ higher, respectively - as illustrated in Table 3.

\section{Toxicities and morbidities}

Radiation-related acute morbidities were evaluated while referring to the CTCAE v5.0 toxicity evaluation protocol. In this study, all the toxicities were of Grade 1-2, and no Grade 3-4 toxicities were reported. As demonstrated by Table 4, the most recurrent morbidity across the investigated patient population was patch mucositis with a rate of $50 \%$. Conversely, the least recurrent morbidity was faint/dull erythema (5\%). 
Table 2. VMAT-based TBI planning parameters and dosimetric coverage

\begin{tabular}{|c|c|c|c|c|c|c|c|c|c|}
\hline \multirow[t]{2}{*}{ Patient No. } & \multicolumn{3}{|c|}{ Planning parameters } & \multicolumn{6}{|c|}{ Dosimetric coverage } \\
\hline & $\begin{array}{l}\text { Single } \\
\text { dose } \\
\text { [Gy] }\end{array}$ & $\begin{array}{l}\text { Fractions } \\
(\#)\end{array}$ & $\begin{array}{l}\text { Total } \\
\text { dose } \\
\text { [Gy] }\end{array}$ & $\begin{array}{c}\text { Mean } \\
\text { Lungs } \\
\text { [Gy] }\end{array}$ & $\begin{array}{c}\text { Mean } \\
\text { Kidneys } \\
\text { [Gy] }\end{array}$ & $\begin{array}{l}\text { Max. } \\
\text { lens } \\
\text { [Gy] }\end{array}$ & $\begin{array}{c}\text { PTV } \\
\text { Mean } \\
\text { [Gy] }\end{array}$ & $\begin{array}{l}\text { PTV } \\
\text { D95 } \\
\text { [Gy] }\end{array}$ & $\begin{array}{c}\mathrm{HI} \\
\text { (D5/D95) }\end{array}$ \\
\hline 1 & 2 & 6 & 12 & 7.82 & 7.14 & 3.66 & 12.63 & 11.4 & 1.11 \\
\hline 2 & 2 & 6 & 12 & 7.72 & 7.24 & 3.99 & 12.55 & 11.5 & 1.12 \\
\hline 3 & 2 & 6 & 12 & 7.21 & 7.16 & 3.83 & 12.43 & 11.4 & 1.13 \\
\hline 4 & 2 & 6 & 12 & 7.72 & 7.91 & 3.96 & 12.77 & 11.5 & 1.16 \\
\hline 5 & 2 & 6 & 12 & 7.88 & 7.94 & 4.53 & 12.64 & 11.5 & 1.18 \\
\hline 6 & 2 & 6 & 12 & 7.91 & 7.56 & 4.79 & 12.93 & 11.3 & 1.22 \\
\hline 7 & 2 & 6 & 12 & 7.68 & 7.62 & 4.88 & 12.51 & 11.3 & 1.13 \\
\hline 8 & 2 & 6 & 12 & 7.93 & 7.82 & 4.01 & 12.41 & 11.4 & 1.11 \\
\hline 9 & 2 & 6 & 12 & 7.69 & 7.91 & 4.39 & 12.75 & 11.5 & 1.15 \\
\hline 10 & 2 & 6 & 12 & 7.82 & 7.96 & 3.94 & 12.53 & 11.5 & 1.11 \\
\hline 11 & 2 & 6 & 12 & 7.65 & 7.72 & 4.53 & 12.71 & 11.3 & 1.14 \\
\hline 12 & 2 & 6 & 12 & 7.83 & 7.93 & 4.72 & 12.86 & 11.5 & 1.16 \\
\hline 13 & 2 & 6 & 12 & 7.55 & 7.63 & 4.11 & 12.63 & 11.4 & 1.14 \\
\hline 14 & 2 & 6 & 12 & 7.92 & 7.49 & 4.92 & 12.83 & 11.5 & 1.15 \\
\hline 15 & 2 & 6 & 12 & 7.78 & 7.71 & 4.16 & 12.92 & 11.3 & 1.16 \\
\hline 16 & 2 & 6 & 12 & 7.58 & 7.33 & 4.61 & 12.76 & 11.4 & 1.17 \\
\hline 17 & 2 & 6 & 12 & 7.93 & 7.72 & 4.41 & 12.58 & 11.3 & 1.12 \\
\hline 18 & 2 & 6 & 12 & 7.95 & 7.88 & 4.81 & 12.97 & 11.5 & 1.21 \\
\hline 19 & 2 & 6 & 12 & 7.86 & 7.68 & 4.61 & 12.61 & 11.4 & 1.16 \\
\hline 20 & 2 & 6 & 12 & 7.79 & 7.51 & 4.91 & 12.88 & 11.3 & 1.17 \\
\hline 21 & 2 & 6 & 12 & 7.42 & 7.38 & 4.74 & 12.62 & 11.5 & 1.12 \\
\hline 22 & 2 & 6 & 12 & 7.76 & 7.55 & 4.44 & 12.66 & 11.4 & 1.21 \\
\hline \multicolumn{4}{|l|}{ Average } & 7.75 & 7.63 & 4.41 & 12.69 & 11.41 & 1.15 \\
\hline \multicolumn{4}{|l|}{ Error } & 0.18 & 0.26 & 0.39 & 0.16 & 0.08 & 0.03 \\
\hline \multicolumn{4}{|l|}{$\%$ Error } & 2.35 & 3.37 & 8.80 & 1.27 & 0.73 & 2.61 \\
\hline \multicolumn{4}{|l|}{ Range } & $7.21-7.95$ & $7.14-7.96$ & $3.66-4.92$ & $12.41-12.97$ & $11.3-11.5$ & $1.11-1.22$ \\
\hline
\end{tabular}

PTV — planned target volume, $\mathrm{HI}$ - heterogeneity index

\section{Discussion}

Linac-based VMAT-TBI has been adopted in numerous studies $[3,8,10]$ owing to some of its advantages, including the highly accurate on-board positioning and intra-fraction motion detecting mechanisms employing $\mathrm{kV} \mathrm{CBCT}$ and optical surface-guided therapy [8]: when compared to helical tomotherapy (HT) and classical TBI. Because secondary malignancies are more prevalent in the 0-10 years age group [15], the benefits of implementing different VMAT-TBI techniques could be manipulated to offer better treatment optimization and organ sparing in pediatric patients.
In recent work by Blomain and colleagues [10], VMAT-TBI pediatric patient plans demonstrated improved gonadal sparing in comparison to simulated two-dimensional (2D) conventional plans. For instance, while the global testes D max. is 2.35 Gy (117.5\%), Blomain et al. [10] reported an improved VMAT-TBI PTV coverage of 2 Gy D90\%, yet that of the conventional 2D anterior-posterior/posterior-anterior (AP-PA) plans was 1.9 Gy D90\% [10]. Following the standard conditioning therapy in almost all allogeneic transplantation centers worldwide, our conditioning regimen implemented a planned total dose of 12 Gy because the transplants were from related donors [3]. Simi- 


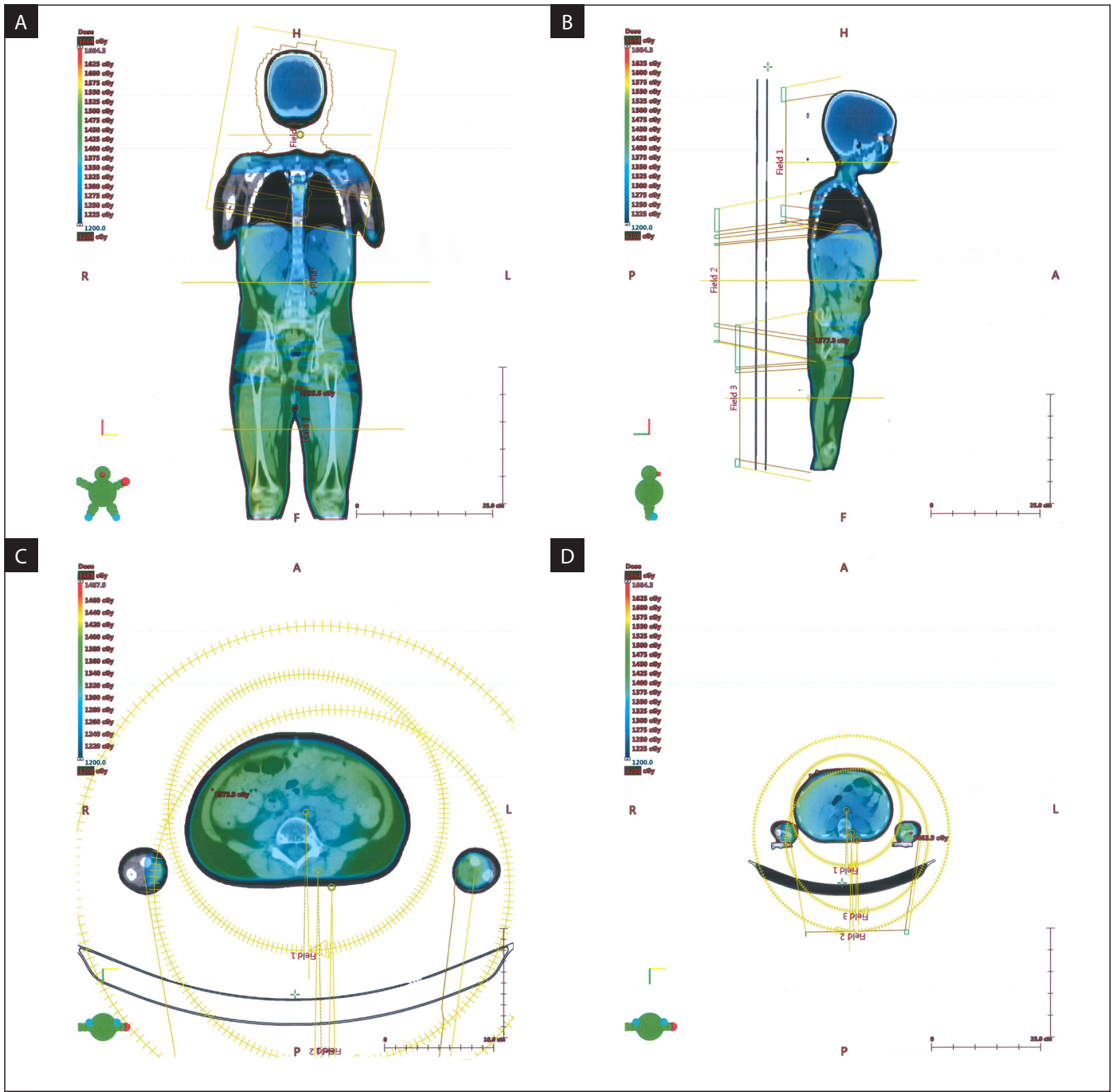

Figure 3. VMAT-based TBI dose distribution in the $(\mathbf{A})$ coronal, $(\mathbf{B})$ sagittal and $(\mathbf{C})$ transverse planes

lar to the work by Blomain et al. [10], our pediatric VMAT-TBI favorably achieved an 11.4 Gy D95\% PTV coverage.

With an age group of 4-22 years, Gruen's group [11] demonstrated HT-TBI as a feasible, well tolerated, and beneficial tool with regard to dose distribution (homogeneity) and reducing doses to OARs. Upon comparison, our mean lung dose was $7.75 \mathrm{~Gy}$, which was less than that (9.14 Gy) of Gruen's group [11]. That was because the patient age group in Gruen's group [11] was from 4-22 years (10 years wider than ours), which implied a larger lung volume, hence a higher mean lung dose. Fur- ther, in another study implementing VMAT-TBI [8], a broader age group from 6-63 years-old (implying a larger lung volume) was treated; hence a higher mean lung dose of 9.7 Gy. Therefore, in order to facilitate comparison of OAR pediatric doses from different clinics worldwide, more studies have to be carried out implementing the same pediatric age group and different VMAT-TBI techniques. Ultimately, a more feasible technique providing better organ sparing doses, while simultaneously achieving the core objectives of TBI, could be attained for pediatric patients. In other terms, one technique could consequently illustrate 
Table 3. Deviation of longitudinal misalignment

\begin{tabular}{|c|c|c|c|c|c|c|}
\hline \multirow{2}{*}{$\begin{array}{l}\text { Patient } \\
\text { No.(\#) }\end{array}$} & \multicolumn{3}{|c|}{ 5-mm misalignment } & \multicolumn{3}{|c|}{ 10-mm misalignment } \\
\hline & $\begin{array}{l}\text { Lung } \\
\text { Mean } \\
\text { (\%) }\end{array}$ & $\begin{array}{l}\text { Lung } \\
\text { Max. } \\
(\%)\end{array}$ & $\begin{array}{c}\text { PTV } \\
\text { Max. } \\
(\%)\end{array}$ & $\begin{array}{l}\text { Lung } \\
\text { Mean } \\
(\%)\end{array}$ & $\begin{array}{l}\text { Lung } \\
\text { Max. } \\
(\%)\end{array}$ & $\begin{array}{c}\text { PTV } \\
\text { Max. } \\
(\%)\end{array}$ \\
\hline 1 & 4.7 & 9.3 & 12.9 & 6.1 & 16.8 & 16.2 \\
\hline 2 & 3.8 & 9.9 & 13.6 & 8.3 & 19.8 & 15.9 \\
\hline 3 & 4.5 & 12.7 & 14.3 & 7.1 & 12.1 & 19.8 \\
\hline 4 & 3.6 & 6.7 & 11.5 & 4.8 & 11 & 18.7 \\
\hline 5 & 4 & 14.1 & 12.9 & 3.9 & 10.3 & 21.1 \\
\hline 6 & 9.5 & 14.5 & 11.8 & 4.1 & 11.4 & 19.2 \\
\hline 7 & 2.9 & 8.8 & 13.2 & 6.4 & 16.2 & 20.6 \\
\hline 8 & 4.4 & 11.5 & 8.9 & 5.1 & 20.7 & 16.4 \\
\hline 9 & 3.5 & 7.6 & 10.5 & 3.9 & 16.7 & 19.3 \\
\hline 10 & 5 & 14.5 & 12.9 & 6.6 & 20.1 & 17.9 \\
\hline 11 & 1.4 & 11.3 & 11.1 & 8.4 & 14.2 & 22.6 \\
\hline 12 & 4.4 & 14.9 & 10.3 & 9.2 & 12.9 & 25.3 \\
\hline 13 & 3.1 & 6.9 & 13.1 & 4.1 & 18.3 & 16.3 \\
\hline 14 & 4.2 & 9.5 & 14.1 & 7.6 & 11.9 & 19 \\
\hline 15 & 4.7 & 8.8 & 11.3 & 7.2 & 13.1 & 17.6 \\
\hline 16 & 1.7 & 5.2 & 11.9 & 6.8 & 8.8 & 14.4 \\
\hline 17 & 4.4 & 7.9 & 16.2 & 3.6 & 16.7 & 20.1 \\
\hline 18 & 2.6 & 9.5 & 13.9 & 6.6 & 13.9 & 16.8 \\
\hline 19 & 3.6 & 7.6 & 14.2 & 5.2 & 23.1 & 15.9 \\
\hline 20 & 5.2 & 4.2 & 10.9 & 6.1 & 16.8 & 16.4 \\
\hline 21 & 4.8 & 9.9 & 12.7 & 7.9 & 11.3 & 22.3 \\
\hline 22 & 2.6 & 13.2 & 7.9 & 8.1 & 13.4 & 21.3 \\
\hline Mean \pm SD & $4.0 \pm 1.6$ & $9.9 \pm 3.1$ & $12.3 \pm 1.9$ & $6.2 \pm 1.7$ & $15.0 \pm 3.8$ & $18.8 \pm 2.7$ \\
\hline
\end{tabular}

PTV — planned target volume; SD — standard deviation

Table 4. Acute radiation morbidity (toxicity)

\begin{tabular}{|l|c|c|}
\hline Grade 1-2 & \multicolumn{1}{c}{$\begin{array}{c}\text { Number } \\
\text { of patients }\end{array}$} & $\begin{array}{c}\text { Percentage } \\
\text { of patients (\%) }\end{array}$ \\
\hline Nausea & 6 & 27 \\
\hline Fatigue & 7 & 32 \\
\hline Mucositis & 11 & 50 \\
\hline Headache & 5 & 23 \\
\hline Loss of appetite & 3 & 14 \\
\hline Xerostomia & 2 & 9 \\
\hline Neck pain & 2 & 9 \\
\hline Erythema & 1 & 5 \\
\hline
\end{tabular}

Toxicity scoring was executed as per CTCAE v5.0, and no Grade 3-4 toxicities were recorded

a better dosimetric characterization for TBI in pediatric patients.
Excluding intentionally shielded or boosted regions, achievement of relative dose homogeneity throughout the body is one of the core objectives in TBI [16]. Similar to elsewhere [8, $17,18]$, we defined $\mathrm{HI}$ as D5/D95 in the present study. A lower HI value (closer to 1) would imply a better dose homogeneity [18]. In our study we reported an average HI value of 1.15 with a standard deviation of 0.03 which, therefore, represents a homogeneous dose distribution in all the patient plans.

Studies exploiting VMAT-TBI have executed various dose verification methods and devices, including ionization chambers [11], films [19], thermoluminescent detectors (TLDs) [11], and optically stimulated luminescent dosimeters (OSLs) [10]. Nevertheless, all these methods hardly cover 
the whole of the comparatively large PTV sizes associated with TBI. Furthermore, they offer 2D dose distributions, yet 3D dose distribution calculations are necessary to facilitate more accurate QA results. That said, we examined dose distributions using the $\mathrm{ArcCHECK}^{\oplus} 4 \mathrm{D}$ detector array with a cylindrical geometry that is designed specifically for rotational delivery. Hence, we executed 3D patient QA by calculating the dose differences between the planned and measured mean doses for the lungs, kidneys and PTV.

The EPID is fundamentally a visualization and patient set-up monitoring system. However, studies have also presented amorphous silicon $(\mathrm{aSi})$ EPIDs as potential detectors and dosimeters [20]. Applying the EPID in a dosimeter mode involves comparison between a calculated fluence map (predicted dose by the TPS) and the real fluency map (measured by the EPID). A gamma analysis tool is used to indicate the calculated-measured dose difference relative to acceptance tolerances [21]. For the $3 \%-3 \mathrm{~mm}$ analysis, the EPID average PTV $\gamma$-index for all the patients was 0.37; that was lower than that (0.39) reported in Tas et al. [8]. In agreement, elsewhere, an EPID-based pre-treatment IMRT verification technique was also demonstrated as a good quality assurance (QA) procedure [20]. Therefore, patient quality assurance was successfully implemented in this study.

Pertaining to toxicity and follow-up, as reported by Wong et al. [2] from Keane et al. [22], the most prevalent toxic effect associated with single large-dose TBI of 8-10 Gy is radiation-induced interstitial pneumonitis, occurring at a $50 \%$ rate of which $50 \%$ of the cases are fatal. In our study, the alternative VMAT-TBI implemented for pediatric patients was associated with mucositis, which occurred in $50 \%$ of the patients in this study. All the acute toxicities reported here were transient and manageable, as suggested by Wong et al. [2]. Even though children are at a higher risk of developing radiation-induced multiple endocrine disorders $[12,23]$, the VMAT-TBI in our study did not induce any of such disorders; as observed during the 9-month follow-up period. Also, similar to the study by Gruen et al. [11], we did not record any grade 3-4 toxicities. Hence, the VMAT-TBI suggested here is a promising and less toxic alternative TBI tool for pediatric patients.

\section{Conclusions}

In our study, we investigated the application of the VMAT-based TBI conditioning regimen in 22 pediatric patients in the $2-12$ years age group. VMAT-based TBI has been presented with feasible dosimetric values in pediatric patients. We obtained a good PTV dose coverage (D95), a low heterogeneity index (homogeneous dose distribution), and the planned TPS doses agreed to those measured by the detector array. After delivery of VMAT-TBI to our pediatric patients, as per the CTCAE v5.0, no grade 3-4 toxicities were observed; only grade 1-2 toxicities were reported. Hence, VMAT-TBI has been presented with a potential of delivering optimum doses to the PTV (whole body) while sparing the sparing OARs in pediatric patients. In the present study, we exploited the dual-arc VMAT-TBI. However, other VMAT-TBI dosimetric characterizations in pediatric patients could be explored using other VMAT techniques such as single-arc and 2-field VMAT for each isocenter.

\section{Conflict of interest}

The authors declare that they have no known competing financial interests or personal relationships that could have appeared to influence the work reported in this paper.

None declared.

\section{Funding}

\section{Acknowledgements}

The authors express their sincere gratitude towards the administration of M.K. Dedeman Oncology Hospital for approving the execution of this study.

\section{Ethical approval}

This article is based on fully anonymized treatment planning data and does not contain any studies with human participants or animals performed by any of the authors.

\section{References}

1. Wong JY, Hui SK. Total Marrow Irradiation. Springer, London 2020.

2. Wong JYC, Filippi AR, Dabaja BS, et al. Total Body Irradiation: Guidelines from the International Lymphoma Radiation Oncology Group (ILROG). Int J Radiat Oncol Biol Phys. 
2018; 101(3): 521-529, doi: 10.1016/j.jjrobp.2018.04.071, indexed in Pubmed: 29893272.

3. Springer A, Hammer J, Winkler E, et al. Total body irradiation with volumetric modulated arc therapy: Dosimetric data and first clinical experience. Radiat Oncol. 2016; 11: 46, doi: 10.1186/s13014-016-0625-7, indexed in Pubmed: 27000180.

4. Mohty M, Malard F, Savani BN, et al. High-dose total body irradiation and myeloablative conditioning before allogeneic hematopoietic cell transplantation: time to rethink? Biol Blood Marrow Transplant. 2015; 21(4): 620-624, doi: 10.1016/j.bbmt.2014.09.010, indexed in Pubmed: 25246296.

5. Hong S, Barker C, Klein JP, et al. Trends in Utilization of Total Body Irradiation (TBI) Prior to Hematopoietic Cell Transplantation (HCT) Worldwide. Biol Blood Marrow Transpl. 2012; 18(2): S336-S337, doi: 10.1016/j.bbmt.2011.12.348.

6. Linsenmeier L, Thoennessen D, Negretti L. Total body irradiation (TBI) in pediatric patients. A single-center experience after 30 years of low-dose rate irradiation. Strahlentherapie und Onkol. 2010; 186(11):614-620, doi: 10.1007/ s00066-010-2089-2, indexed in Pubmed: 21069270.

7. Bölling $T$, Kreuziger DC, Ernst I, et al. Retrospective, monocentric analysis of late effects after Total Body Irradiation (TBI) in adults. Strahlenther Onkol. 2011; 187(5): 311-315, doi: 10.1007/s00066-011-2190-1, indexed in Pubmed: 21533759.

8. Tas B, Durmus IF, Okumus A, et al. Total-body irradiation using linac-based volumetric modulated arc therapy: Its clinical accuracy, feasibility and reliability. Radiother Oncol. 2018; 129(3): 527-533, doi: 10.1016/j.radonc.2018.08.005, indexed in Pubmed: 30172456.

9. Symons K, Morrison C, Parry J, et al. Volumetric modulated arc therapy for total body irradiation: A feasibility study using Pinnacle treatment planning system and Elekta Agility $^{\mathrm{TM}}$ linac. J Appl Clin Med Phys. 2018; 19(2): 103-110, doi: 10.1002/acm2.12257, indexed in Pubmed: 29368389.

10. Blomain ES, Kovalchuk N, Neilsen BK, et al. A Preliminary Report of Gonadal-Sparing TBI Using a VMAT Technique. Pract Radiat Oncol. 2021; 11(2): e134-e138, doi: 10.1016/j. prro.2020.07.006, indexed in Pubmed: 32795616.

11. Gruen A, Ebell W, Wlodarczyk W, et al. Total Body Irradiation (TBI) using Helical Tomotherapy in children and young adults undergoing stem cell transplantation. Radiat Oncol. 2013; 8: 92, doi: 10.1186/1748-717X-8-92, indexed in Pubmed: 23587349.

12. Ricardi U, Filippi AR, Biasin E, et al. Late toxicity in children undergoing hematopoietic stem cell transplantation with TBI-containing conditioning regimens for hematological malignancies. Strahlenther Onkol. 2009; 185 Suppl 2:
17-20, doi: 10.1007/s00066-009-1008-x, indexed in Pubmed: 19685029.

13. Ouyang L, Folkerts $M$, Zhang $Y$, et al. Volumetric modulated arc therapy based total body irradiation: Workflow and clinical experience with an indexed rotational immobilization system. Phys Imag Radiat Oncol. 2017; 4: 22-25, doi: 10.1016/j.phro.2017.11.002.

14. Losert C, Shpani R, Kießling R, et al. Novel rotatable tabletop for total-body irradiation using a linac-based VMAT technique. Radiat Oncol. 2019; 14(1): 244, doi: 10.1186/ s13014-019-1445-3, indexed in Pubmed: 31888680.

15. Council R. Health risks from exposure to low levels of ionizing radiation: BEIR VII phase 2. Vol. 7. National Academies Press, Washington 2006.

16. Achari R, Das A, Mahata A. Total Body Irradiation in Stem Cell Transplant. Contemp Bone Marrow Transplant. 2020: 1-18, doi: 10.1007/978-3-319-64938-2_23-1.

17. Kataria T, Sharma K, Subramani V, et al. Homogeneity Index: An objective tool for assessment of conformal radiation treatments. J Med Phys. 2012; 37(4): 207-213, doi: 10.4103/0971-6203.103606, indexed in Pubmed: 23293452.

18. Gong Y, Wang J, Bai S, et al. Conventionally-fractionated image-guided intensity modulated radiotherapy (IGIMRT): a safe and effective treatment for cancer spinal metastasis. Radiat Oncol. 2008; 3: 11, doi: 10.1186/1748717X-3-11, indexed in Pubmed: 18426607.

19. Zhuang AH, Liu An, Schultheiss TE, et al. Dosimetric study and verification of total body irradiation using helical tomotherapy and its comparison to extended SSD technique. Med Dosim. 2010; 35(4): 243-249, doi: 10.1016/j. meddos.2009.07.001, indexed in Pubmed: 19944588.

20. Grządziel A, Smolińska B, Rutkowski R, et al. EPID dosimetry - configuration and pre-treatment IMRT verification. Rep Pract Oncol Radiother. 2007; 12(6): 307-312, doi: 10.1016/s1507-1367(10)60069-7.

21. Low DA, Harms WB, Mutic $S$, et al. A technique for the quantitative evaluation of dose distributions. Med Phys. 1998; 25(5): 656-661, doi: 10.1118/1.598248, indexed in Pubmed: 9608475.

22. Keane TJ, Dyk Jv, Rider WD. Idiopathic interstitial pneumonia following bone marrow transplantation: The relationship with total body irradiation. Int J Radiat Oncol Biol Phys. 1981; 7(10): 1365-1370, doi: 10.1016/03603016(81)90032-8, indexed in Pubmed: 7033190.

23. Ishida Y, Sakamoto N, Kamibeppu K, et al. Late effects and quality of life of childhood cancer survivors: Part 2. Impact of radiotherapy. Int J Hematol. 2010; 92(1): 95-104, doi: 10.1007/s12185-010-0611-z, indexed in Pubmed: 20577841. 